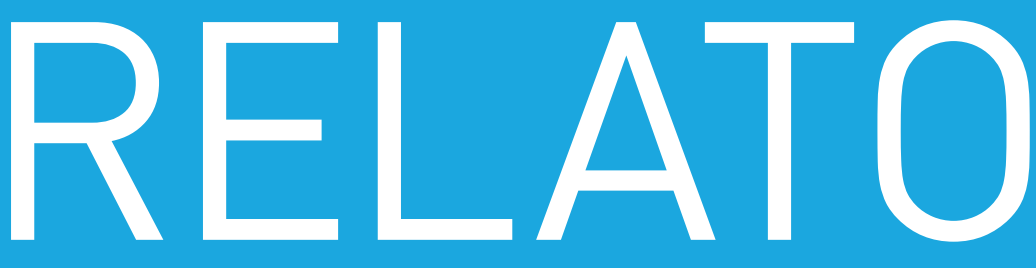

\title{
ARIPIPRAZOL NA SÍNDROME DE TOURETTE EM ADULTOS: RELATO DE CASO E REVISÃO NARRATIVA
}

\section{ARIPIPRAZOLE IN TOURETTE'S SYNDROME IN ADULTS: CASE REPORT AND NARRATIVE REVIEW}

\section{Resumo}

A síndrome de Tourette é um distúrbio de movimento caracterizado por tiques motores e vocais que surgem na infância e na adolescência, com metade a dois terços dos casos remitindo ainda nessas fases. Persistindo mais raramente na idade adulta, apresenta um prognóstico reservado, com difícil controle dos sintomas. $O$ presente artigo descreve o uso de aripiprazol no tratamento da síndrome de Tourette em um adulto do sexo masculino e faz uma revisão narrativa de literatura sobre as evidências atuais disponíveis com o uso desse medicamento.

Palavras-chave: Síndrome de Tourette, aripiprazol, tratamento.

\section{Abstract}

Tourette's syndrome is a movement disordercharacterized by motor and vocal tics that arise in childhood and adolescence; half to two-thirds of the cases remit still in these stages. The disorder is more rarely found in adulthood, usually having a guarded prognosis and symptoms that are difficult to control in this population. This article describes the use of aripiprazole for the treatment of Tourette's syndrome in a young male adult and conducts a narrative literature review of the evidence currently available on the use of this medication.

Keywords: Tourette's syndrome, aripiprazole, treatment.

\section{INTRODUÇÃO}

A síndrome de Tourette (ST) é uma desordem neuropsiquiátrica que afeta crianças e adultos $^{1}$. Caracterizada pelo aparecimento de tiques súbitos/ rápidos, movimentos motores recorrentes e/ou vocalizações, ela pode afetar negativamente a qualidade de vida de seus portadores ${ }^{2}$. Os estudos também mostram ser mais frequente a presença de transtornos comórbidos (depressão, ansiedade, transtorno obsessivo-compulsivo, déficit de atenção e hiperatividade) na síndrome ${ }^{3}$.

A hipótese de que os tiques são causados por problemas do circuito motor e do sistema dopaminérgico levou os antipsicóticos a serem considerados o tratamento principal ${ }^{4}$. Estudos controlados em crianças e adolescentes mostram diminuição da frequência de tiques em 50\% após o uso de haloperidol e pimozida. Entretanto, devido ao perfil de maiores efeitos extrapiramidais, os antipsicóticos típicos passam a ser pouco tolerados no longo prazo. Os antipsicóticos atípicos, apesar de apresentarem menos sintomas extrapiramidais, ainda chegam a ter efeitos indesejáveis em até $80 \%$ dos pacientes ${ }^{5}$. Maior propensão a ganho de peso, sedação, hipotensão ortostática e efeitos metabólicos adversos, como diabetes tipo II e doenças cardiovasculares, tornam a prescrição dos neurolépticos de segunda geração mais restritab.

O aripiprazol é um antipsicótico atípico de funcionamento particular. Seu mecanismo de ação tem como base o agonismo parcial dos receptores D2, 5-HT2C e 5-HT1A, além do antagonismo do receptor 5-HT2A, o que provavelmente explica a sua eficácia em casos refratários e a ocorrência menor de efeitos colaterais $^{7,8}$.

\section{RELATO dE CASO}

Paciente masculino, 34 anos, desempregado, iniciou acompanhamento no ambulatório de psiquiatria do 


\section{KARINA FARIAS FERRAZ', HENRIQUE TOSCANO SIEBRA BRITO², LEOPOLDO NELSON DE FERNANDES BARBOSA ${ }^{3}$, HENRIQUE FARIA DE SOUSA ${ }^{4}$}

Médica residente de Psiquiatria, Instituto de Medicina Integral Professor Fernando Figueira (IMIP), Recife, PE. ${ }^{2}$ Médico residente de Psiquiatria, IMIP, Recife, PE. ${ }^{3}$ Psicólogo e preceptor de Saúde Mental, IMIP, Recife, PE. ${ }^{4}$ Coordenador, Serviço de Psiquiatria, e Preceptor, Residência Médica em Psiquiatria, IMIP, Recife, PE.

Instituto de Medicina Integral Professor Fernando Figueira (IMIP), em Recife (PE), em maio de 2017. Relatava que, entre 3 e 5 anos de idade, passou a apresentar piscadelas, caretas, tiques motores simples em pescoço, membros superiores e inferiores, coprolalias, ecolalia e emissão de sons semelhantes a latidos. Há 7 anos, iniciou acompanhamento no ambulatório de psiquiatria de outro serviço, onde foi diagnosticado com ST. Foram prescritos risperidona $2 \mathrm{mg} 2 \times /$ dia e cloxazolam $2 \mathrm{mg} 2 \times /$ dia, com melhora parcial. Posteriormente, perdeu seguimento ambulatorial no antigo serviço.

Foi reiniciado tratamento no serviço do IMIP. Em primeira consulta, referia tristeza, desmotivação, inapetência, insônia e dores musculares. Apresentavase com humor deprimido e discurso de conteúdo de menos-valia e desesperança. Apertava os olhos, fungava, flexionava o pescoço e fazia movimentos de socar com os braços. Foram reiniciados risperidona 2 mg 2×/dia, cloxazolam $2 \mathrm{mg}$, sendo meio comprimido pela manhã e um comprimido à noite, e escitalopram $10 \mathrm{mg} /$ dia. Paciente evoluiu com melhora do humor deprimido. Ainda apresentava movimentos de flexão e rotação interna de antebraços, piscadelas, fazia bico e sons semelhantes a latidos.

Aumentou-se risperidona a $3 \mathrm{mg} 2 \times / \mathrm{dia}$, e diminuiuse cloxazolam a $2 \mathrm{mg} / \mathrm{dia}$. Escitalopram $10 \mathrm{mg} / \mathrm{dia}$ foi mantido. O paciente apresentou tontura e sensação de desmaio. Permaneceu com tiques motores e vocais. Foi feito desmame de risperidona e iniciado aripiprazol $10 \mathrm{mg} /$ dia. Alcançou ausência de tiques em membros e pescoço, manteve piscadelas e tiques vocais com menor frequência. Foi aumentado aripiprazol a 15 $\mathrm{mg} /$ dia. Substituiu-se antidepressivo por sertralina 50 $\mathrm{mg} / \mathrm{dia}$, pois se queixava de diminuição da libido com escitalopram. Foi observada melhora de libido, porém evoluiu com humor ansioso. Optou-se por aumentar sertralina a $75 \mathrm{mg} / \mathrm{dia}$.

Em consulta seguinte, após dois meses, foi observada melhora de humor, contudo o paciente ainda apresentava tiques na face do tipo piscadelas e fungado. Foi aumentado aripiprazol para $20 \mathrm{mg} / \mathrm{dia}$, sendo observada ausência de tiques durante reavaliação após quatro meses e manutenção da melhora do humor. Paciente voltou a exercer atividade laboral como carregador em empresa privada e a ter maior sociabilização com familiares e amigos do local de trabalho.

\section{DIsCUSSÃo}

Em uma série de casos $^{9}, 100$ pacientes com idade média de 27 anos ( \pm 11 ) foram tratados com doses diárias de aripiprazol que variavam de 5 a $45 \mathrm{mg}$. Noventa e cinco desses pacientes haviam feito tratamentos anteriores malsucedidos com um ou mais neurolépticos. 0 resultado desse estudo apontou que 82 pacientes exibiram redução considerável dos tiques e que, em 48 pacientes, a efetividade do tratamento se estendeu por mais de 12 meses.

Outro estudo publicado na Turquia ${ }^{10}$ relatou importante diminuição dos tiques e menos efeitos colaterais em oito casos de pacientes com idade entre 9 e 57 anos tratados com aripiprazol. Todos os pacientes relataram ensaios prévios com outros antipsicóticos e interrupção do tratamento devido aos efeitos adversos. Uma metanálise realizada na China ${ }^{11}$ abrangeu 22 revisões sistemáticas sobre o tratamento farmacológico e comportamental de tiques. Três desses estudos indicaram que haloperidol e pimozida (antipsicóticos típicos) foram eficazes na redução dos tiques em comparação ao placebo, porém apresentaram baixa tolerabilidade. Seis outros estudos avaliaram a eficácia de antipsicóticos atípicos e indicaram que risperidona e aripiprazol melhoraram significativamente os sintomas de tiques em comparação ao placebo ou antipsicóticos típicos, com menos efeitos colaterais.

Uma revisão sistemática sobre o uso do aripiprazol no tratamento do transtorno de tiques em crianças e adolescentes $^{12}$ analisou 35 artigos, sendo que a maioria eram relatos de caso e apenas dois eram ensaios com grupo-controle. Não havia ensaios clínicos duplo-cegos e, portanto, faltavam evidências fortes quanto ao uso do aripiprazol. Entretanto, esse estudo sugeria a eficácia do aripiprazol nos distúrbios de tiques, além do perfil mais seguro da droga comparado ao dos neurolépticos típicos.

Outra metanálise realizada ${ }^{13}$ não encontrou diferença significativa na redução dos tiques entre indivíduos tratados com aripiprazol, haloperidol ou tiapride. Quatro estudos que compararam aripiprazol ao haloperidol não revelaram diferença significativa na redução dos 
tiques. Da mesma forma, dois estudos que compararam aripiprazol a tiapride não mostraram diferença significativa na redução dos tiques. Os autores concluíram que aripiprazol parecia ser uma terapia promissora para crianças com transtornos de tiques.

A maioria dos trabalhos sobre o tratamento da ST com aripiprazol envolve relatos de caso e estudos abertos com crianças e adolescentes. Não há ensaios controlados nem séries de casos que incluam um número substancial de pacientes adultos. Somado a isso, os dados sobre tratamento com aripiprazol em longo prazo são escassos?.

\section{CONCLUSÃO}

A ST é um transtorno neuropsiquiátrico com implicações sociais, sendo importante o diagnóstico na infância ${ }^{14}$. Diante dos antipsicóticos, o aripiprazol parece ter um papel importante no tratamento da ST - fato talvez relacionado ao agonismo parcial de D2. Porém, ainda são necessários estudos mais robustos para consolidar o tratamento com aripiprazol ${ }^{15}$.

Artigo submetido em 16/08/2019, aceito em 23/08/2019. Os autores informam não haver conflitos de interesse associados à publicação deste artigo.

Fontes de financiamento inexistentes.

Correspondência: Karina Farias Ferraz, Rua Deputado Cunha Rabelo, 219/302, Várzea, CEP 50740-400, Recife, PE. E-mail: kafafe13@gmail.com

\section{Referências}

1. Janik P, Szejko N. Aripiprazole in treatment of Gilles de la Tourette syndrome - new therapeutic option. Neurol Neurochir Pol. 2018;52:84-7.

2. Sallee F, Kohegyi E, Zhao J, McQuade R, Cox K, Sanchez R, et al. Randomized, double-blind, placebo-controlled trial demonstrates the efficacy and safety of oral aripiprazole for the treatment of Tourette's disorder in children and adolescents. J Child Adolesc Psychopharmacol. 2017;27:771-81.

3. Gerasch S, Kanaan AS, Jakubovski E, Müller-Vahl KR. Aripiprazole improves associated comorbid conditions in addition to tics in adult patients with Gilles de la Tourette syndrome. Front Neurosci. 2016;10:416.
4. Huys D, Hardenacke K, Poppe P, Bartsch C, Baskin B, Kuhn J. Update on the role of antipsychotics in the treatment of Tourette syndrome. Neuropsychiatr Dis Treat. 2012;8:95104.

5. Lavoie ME, O'Connor K. Toward a multifactorial conception of the Gilles de la Tourette syndrome and persistent chronic tic disorder. Brain Sci. 2017 Jun 2;7(6). pii: E61.

6. Budman CL. The role of atypical antipsychotics for treatment of Tourette's syndrome: an overview. Drugs. 2014;74:1177-93.

7. Bhatia M, Gautam P, Kaur J. Case reporton Tourette syndrome treated successfully with aripiprazole. Shanghai Arch Psychiatry. 2014;26:297-9.

8. Zheng W, Li XB, Xiang YQ, Zhong BL, Chiu HF, Ungvari GS, et al. Aripiprazole for Tourette's syndrome: a systematic review and meta-analysis. Hum Psychopharmacol. 2016;31:11-8.

9. Wenzel C, Kleimann A, Bokemeyer S, Müller-Vahl KR. Aripiprazole for the treatment of Tourette syndrome: a case series of 100 patients. J Clin Psychopharmacol. 2012;32:548-50.

10. Taskiran S, Tufan AE, Semerci B. Aripiprazole can a viable choice for persistant suppression of symptoms in managing chronic tic disorders and Tourette's disorder through the life span: a case series. KlinikPsikofarmakol Bülteni. 2016;26:181-5.

11. Yang C, Hao Z, Zhu C, Guo Q, Mu D, Zhang L. Interventions for tic disorders: an overview of systematic reviews and meta analyses. Neurosci Biobehav Rev. 2016;63:239-55.

12. Ghanizadeh A. Systemic review of aripiprazole for the treatment of children and adolescents with tic disorders. Neurosciences(Riyadh). 2012;17:200-4.

13. Yang CS, Huang H, Zhang LL, Zhu CR, Guo Q. Aripiprazole for the treatment of tic disorders in children: a systematic review and meta-analysis. BMC Psychiatry. 2015;15:179.

14. Eddy CM, Rickards HE, Cavanna AE. Treatment strategies for tics in Tourette syndrome. Ther Adv Neurol Disord. 2011;4:25-45.

15. Padala PR, Qadri SF, Madaan V. Aripiprazole for the treatment of Tourette's disorder. Prim Care Companion J Clin Psychiatry. 2005;7:296-9. 